ACCEPTED MANUSCRIPT

\title{
Measuring magnetocaloric effect in a phase separated system
}

To cite this article before publication: Dafne Yael Goijman et al 2018 Mater. Res. Express in press https://doi.org/10.1088/2053-1591/aaf04a

\section{Manuscript version: Accepted Manuscript}

Accepted Manuscript is "the version of the article accepted for publication including all changes made as a result of the peer review process, and which may also include the addition to the article by IOP Publishing of a header, an article ID, a cover sheet and/or an 'Accepted Manuscript' watermark, but excluding any other editing, typesetting or other changes made by IOP Publishing and/or its licensors"

This Accepted Manuscript is @ 2018 IOP Publishing Ltd.

During the embargo period (the 12 month period from the publication of the Version of Record of this article), the Accepted Manuscript is fully protected by copyright and cannot be reused or reposted elsewhere.

As the Version of Record of this article is going to be / has been published on a subscription basis, this Accepted Manuscript is available for reuse under a CC BY-NC-ND 3.0 licence after the 12 month embargo period.

After the embargo period, everyone is permitted to use copy and redistribute this article for non-commercial purposes only, provided that they adhere to all the terms of the licence https://creativecommons.org/licences/by-nc-nd/3.0

Although reasonable endeavours have been taken to obtain all necessary permissions from third parties to include their copyrighted content within this article, their full citation and copyright line may not be present in this Accepted Manuscript version. Before using any content from this article, please refer to the Version of Record on IOPscience once published for full citation and copyright details, as permissions will likely be required. All third party content is fully copyright protected, unless specifically stated otherwise in the figure caption in the Version of Record.

View the article online for updates and enhancements. 


\title{
Measuring magnetocaloric effect in a phase-separated system.
}

\author{
D. Goijman ${ }^{1,4}$, A. G. Leyva ${ }^{2,3,4}$, M. Quintero $2,3,4$ \\ 1- Laboratorio de Resonancias Magnéticas, Gerencia de Física, GAIANN, Centro Atómico Bariloche, Comisión Nacional \\ de Energía Atómica (CNEA), Av Bustillo 9500, Bariloche, Argentina. \\ 2-Departamento de Física de la materia Condensada, GlyAN, GAIANN, Comisión Nacional de Energía Atómica (CNEA), Av. \\ Gral. Paz 1499, San Martin, Buenos Aires, Argentina. \\ 3-Escuela de Ciencia y Tecnología, Universidad Nacional de General San Martin, Alem 3901, San Martin, Buenos Aires, \\ Argentina. \\ 4-Instituto de Nanociencia y Nanotecnología, Av. Gral. Paz 1499, San Martin, Buenos Aires, Argentina.
}

\section{Abstract.}

The magnetocaloric effect (MCE) in a magnetic system can be characterized by the estimation of the entropy change produced when a magnetic field is applied. The discrepancies between the results obtained with different methods have encouraged the scientific community to attempt to better understand the procedures associated with this calculation. Within this context, we present a study about how the presence of an inhomogeneous state influences the determination of the entropy change. To do that we chose a prototypical system used to study the phase separation phenomena in manganites. We compared results obtained using different methods, and we propose an approach to correlate the results obtained following different procedures. 


\section{Introduction}

The magnetocaloric effect (MCE) is the temperature change produced in a magnetic system when an external magnetic field is applied. Even though the effect was discovered in 1917 by Weiss and Picard[1], the discovery of a large MCE at room temperature in Gd (SiGe) [2] was a new starting point in the study of this effect, which triggered the development of solid state cooling devices based on the MCE. Nowadays, the study of MCE has been extended to a large number of systems, namely Gd nanoparticles [3], As based compounds [4], Heusler alloys[5] [6] and manganites [7] [8]among others.

The MCE can be quantified considering the magnetic entropy change $(\Delta S)$ produced by the application of the magnetic field. The entropy change can be obtained from both heat capacity and from magnetization measurements[9], using a Maxwell relation to calculate $\Delta S$ by numerical integration of a set of magnetization curves[10].

As the interest in the MCE expanded to a large number of compounds, the methods used to calculate it were carefully revised, especially in those cases where first order phase transitions were involved [11] [12] [13] [14] [15] . The correct application of the Maxwell relation requires a transition between different equilibrium states [16] [17], and its inappropriate use might lead to an overestimation of the entropy change. In order to avoid these undesired effects, some authors propose the use of alternative methods, based on geometric arguments [18] or the ClasiusClapeyron equation[19]. Another important aspect is the thermodynamic history of the sample. L. Caron et al [16] [20]analyzed the influence of the magnetization measurement protocol in the determination of the entropy change.

Nowadays, despite the advances in the development of direct measurement techniques, the change of entropy is mostly obtained from indirect measurements. Therefore, the study of these techniques is a crucial aspect towards the use of caloric materials for magnetic refrigeration. 
From the large number of systems exhibiting MCE, the mixed valence manganese oxides (manganites) are one of the most promising ones, due to their unique ability to tune their magnetic, electric and structural properties by minor modifications in their compositions or in the synthesis parameters (grain size[21], oxygen stoichometry[22], etc). The strong coupling between the different degrees of freedom in manganites is another aspect to take into account, since it is possible to induce a structural or electrical phase transition by the application of moderate external stimuli (i.e. magnetic field, hydrostatic pressure, radiation).

The coexistence of regions with different magnetic, electrical and structural properties, the so called phase separation (PS) phenomenon, is one of the most widely studied topics in mixed valence manganese oxides. Despite the enormous efforts made by the scientific community, a complete explanation of the phase coexistence origin is still lacking. The out-of-equilibrium nature of this inhomogeneous state leads to a rich variety of behaviors related to the competition between the different phases. Given this scenario $\mathrm{La}_{0.5} \mathrm{Ca}_{0.5} \mathrm{MnO}_{3}(\mathrm{LCMO})$ and $\mathrm{La}_{5 / 8-\mathrm{y}} \mathrm{Pr}_{\mathrm{y}} \mathrm{Ca}_{3 / 8} \mathrm{MnO}_{3}$ (LPCMO) are two prototypical systems where the PS phenomenon has been studied.

In LCMO the PS has been studied using different techniques, such as magnetization[23], neutron powder diffraction[24], and many others[25] [26] [27] [28] [29]. In this system, the ferromagnetic metallic (FM) phase competes with the antiferromagnetic (AFM) phase and charge ordering (CO) one, leading to the presence of a dynamic behavior related to the system evolution towards the equilibrium state. By controlling the grain size, it is possible to tune the fraction of the CO phase, through the frustration of the long range order, which favors the stabilization of the FM state [21], while remaining completely suppressed for grain sizes below $15 \mathrm{~nm}[30]$.

In the case of LCMO, the system can be controlled by modifying the $\mathrm{Pr} /$ La proportion. In the rich $\mathrm{Pr}$ region of the phase diagram, the system is mainly dominated by an AFM and CO phase, while a 
mainly FM state is observed in the La rich region. For Pr content around 0.3 , a strong PS region is present, and has been observed using different techniques[31] [32] [33]. For both systems, the MCE has been studied in order to understand the complex scenario involving the presence of PS in manganites, but also as an example of how the inadequate use of an indirect method can lead to the overestimation of the MCE [34] [35].

Phan et al [36] analyze the evolution of the entropy change caused by the magnetic field in different temperature ranges in LPCMO, revealing a strong correlation between the entropy change and the system's dynamic behavior. Similarly, Amirzadeh et al [37] studied the MCE in LCMO as a function of grain size, obtaining an anomalous behavior that could be associated with the presence of a magneto-structural transition.

In this paper, we will discuss the influence of the PS state in the determination of the MCE in manganites. We will compare the results obtained using different methods (Maxwell's relation and heat capacity) and analyze discrepancies y coincidences. Finally we will introduce the necessary modifications to correct the artifacts introduced when the Maxwell relation is applied on an out-of-equilibrium system.

\section{Methods}

Polycrystalline $\mathrm{La}_{0.625-\mathrm{y}} \mathrm{Pr}_{\mathrm{y}} \mathrm{Ca}_{0.375} \mathrm{MnO}_{3}$ samples were synthesized using the Sol-gel technique and characterized by X-Ray diffraction to confirm composition, structure and to ensure good quality. Magnetic measurements were performed using the vibrating sample magnetometry technique, and heat capacity was measured using adiabatic relaxation in a Versalab system manufactured by Quantum Design. In all the results presented in this paper, magnetization as a function of temperature was measured on cooling with the applied magnetic field and a cooling rate of 1 $\mathrm{K} / \mathrm{min}$. in sweep mode. Isothermal magnetization loops were measured after cooling the sample 
without an applied magnetic field, starting at room temperature (the sample was always warmed up to room temperature between measurements).

Heat capacity measurements were taken on cooling, with a variable cooling rate due to the characteristics of the adiabatic relaxation method, which requires temperature stability.

\section{Results}

The phase diagram for $\mathrm{La}_{0.625-\mathrm{y}} \mathrm{Pr}_{\mathrm{y}} \mathrm{Ca}_{0.375} \mathrm{MnO}_{3}$, schematized in Figure 1, can be divided in three regions, varying in $\operatorname{Pr}$ content $(y)$. In the low y region $(y<0.20)$, the system presents a ferromagnetic metallic (FMM) behavior for temperatures below $\mathrm{Tc} \sim 230 \mathrm{~K}$. The magnetization value obtained for the sample with $y=0.20$ (LPC-20) (fig 2a) is consistent with the complete alignment of the Mn ions. In the magnetization as a function of magnetic field curves it is possible to see how the paramagnetic state give place to the FM state as the temperature decreases.

On the opposite side, for $\mathrm{y}>0.5$ a charge $\operatorname{order}(\mathrm{CO})$ appears at Tco $~ 210 \mathrm{~K}$ on cooling. Some degrees below, the $\mathrm{Mn}$ ions are anti-ferromagnetically ordered (AFM)[38]. The magnetization values obtained for the sample with $y=0.50$ (LPC-50) (fig2 c) are one order of magnitude smaller than the ones observed for the ferromagnetic ordering. The corresponding magnetization as a function of the magnetic field measurements confirms the presences of the mentioned phases.

In the intermediate region, the system is characterized by the presence of the phase separation (PS) state. In figure $2 b$, we present magnetization as a function of temperature for the sample with $y=0.32(\mathrm{LPC}-32))$. The room temperature state of the system is paramagnetic insulator, as can be seen in the linear behavior of the magnetization as a function of magnetic field curves. On cooling, the CO phase appears at Tco $=220 \mathrm{~K}$ and some degrees below, at $\mathrm{Tc}^{\sim} 200 \mathrm{~K}$, the magnetization increases, indicating the formation of FM regions. The presence of an 
inhomogeneous state can be observed in the mangentization as a function of magentic field curves, characterized by a two step increase. The system maintains an almost constant magnetization value, and down to $\mathrm{T}^{\sim} 100 \mathrm{~K}$, where the magnetization values present an abrupt increase, reaching values consistent with a homogeneous FM state at lower temperatures. In order to understand how the behavior described above is reflected in magnetocaloric properties, it is necessary to determine the entropy change. Following the most commonly accepted approach, it is possible to estimate this change using numerical integration of magnetization loops at different temperatures as

$\Delta S_{M}=\frac{1}{\Delta T} \int_{0}^{H}\left[M\left(T+\Delta T, H^{\prime}\right)-M\left(T, H^{\prime}\right)\right] d H^{\prime}$ (1) being $\mathrm{M}$ and $\mathrm{H}$ the magnetization and the applied magnetic field, respectivelly.

In Figure 3 we show the temperature dependence of the entropy change obtained from the $M(H)$ branch measured increasing $\mathrm{H}$ from 0 to $3 \mathrm{~T}$. In order to avoid the "peak effect" usually observed around first order transitions, we warmed up the samples above Curie temperature after each magnetization loop measurement [16].

For LPC-20 and LPC-32 samples, $\mathrm{H}$ is strong enough to suppress the CO phase and leads to a homogeneous FM, in contrast to what happens with LPC-50, where the magnetic field does not convert the CO/AFM phase to FM.

For LPC-20, the entropy displays a peak at $235 \mathrm{~K}$ with a value of $9 \mathrm{~J} / \mathrm{Kg}$. $\mathrm{K}$, which is consistent with the PI to FM transition, and close to the values already reported [8]. The LPC-50 sample presents two peaks, one of $0.3 \mathrm{~J} / \mathrm{Kg} \mathrm{K}$ at $235 \mathrm{~K}$, and another one which is three times smaller, at $170 \mathrm{~K}$. The 
where

$$
\Delta S_{H C}(T, H)=\int_{T_{0}}^{T} \frac{C_{P}\left(T^{\prime}, H\right)-C_{P}\left(T^{\prime}, H=0\right)}{T^{\prime}} d T^{\prime}+\Delta S\left(T_{0}, H\right)
$$

$\mathrm{Cp}(\mathrm{T}, \mathrm{H})$ is the heat capacity at constant pressure

$\Delta S\left(T_{0}, H\right)$ is the difference in zero temperature entropies.

In Figure 4 we show the entropy change $\left(\Delta S_{H C}\right)$ as a function of temperature for samples LPC-20,

LPC-32 and LPC-50 obtained from heat capacity curves. In all the cases, the lowest temperature reached was $50 \mathrm{~K}$.

For LPC-20 and LPC-50, $\Delta S_{H C}(50 K)$ was considered as zero for different reasons. In the first case, the sample is a soft FM and at $50 \mathrm{~K}$ the magnetic moments are completely aligned at zero field. In the second case, the maximum applied $\mathrm{H}$ is not enough to modify the $\mathrm{CO}$ phase.

In the case of LPC-32, the value of $\Delta S_{H C}(50 K)$ was obtained comparing the entropy change in the high temperature paramagnetic phase with the value obtained from $\Delta S_{M}(T)$. The result 
$\Delta S_{H C}(50 K)=-6.74 J / K g K$ is consistent with the values measured during a FM to CO

transition in similar samples[40] [41].

\section{Discussion}

We observe a good agreement between the results obtained using Maxwell's relation and the heat capacity for samples LPC-20 and LPC-50.

Comparing $\Delta S_{H C}(T)$ and $\Delta S_{M}(T)$ for LPC-32, we observed substantial differences below $210 \mathrm{~K}$.

These discrepancies could be related to the use of Maxwell's relation under inadequate conditions, such as the coexistence of two different phases and possible hysteresis losses.

In order to analyze the contribution of each of the abovementioned effects, we proposed the following modifications to the expression for $\Delta$ S obtained using Maxwell's relations.

The first modification takes into account the inhomogeneous state observed in the intermediate region. When the entropy change is calculated for a certain temperature using the numerical integration for equation 1 , the result obtained is mainly due to the magnetization change at the PIFM transition, but it is also affected by the change in the relative fraction of the FM phase (f) [13]. The temperature dependence of $f$ can be extracted from the magnetization as a function of temperature, and it can be initially normalized with the magnetization of the entire $M n$ network aligned with the magnetic field.

Then

$\Delta S_{M O D}(T)=\Delta S_{H}(T)+\alpha \cdot f(T)$

where 
$\Delta S_{M O D}(T)$ is the modified entropy change

$\Delta S_{H}(T)$ is the entropy corresponding to the homogeneous system.

The other contribution to be considered is energy losses due to the presence of hysteresis in the $M(H)$ curves, which can be related to the presence of defects such as grain boundary, tangles of dislocation, precipitates or inhomogeneities that hinder the advance of the domain walls, preventing the system from reaching the global energy state [42].

To analyze the effect of the hysteresis, we will assume that the system's equilibrium state will be a reversible curve named Man in Figure 5. The hysteretic magnetization curve can be separated in two different branches, one obtained when the magnetic field is increased $\left(M_{\uparrow}\right)$ and the other one corresponding to a decrease in the magnetic field $\left(M_{\downarrow}\right)$. For simplicity's sake, we chose for $M_{\text {AN }}$ to be in the center of the hysteresis loop, so we that we could introduce a parameter $(\delta)$ to quantify the hysteresis as:

$$
\begin{aligned}
& M_{\uparrow}=M_{A N}-\delta \\
& M_{\downarrow}=M_{A N}+\delta
\end{aligned}
$$

When the entropy change is calculated using Maxwell's relation, it is usually calculated based on $M_{\uparrow}$ curves. Then, it can be expressed as a function of $M_{A N}$ as:

$$
\Delta S_{M}=\frac{1}{\Delta T} \int_{0}^{H}\left[M_{A N}\left(H^{\prime}, T+\Delta T\right)-\delta\left(H^{\prime}, T+\Delta T\right)-M_{A N}\left(H^{\prime}, T\right)+\delta\left(H^{\prime}, T\right)\right] d H^{`}
$$

Introducing the magnetic work (W) as the area enclosed by $M_{\uparrow}$ and $M_{\downarrow}$, it can be expressed in terms of $\delta$ :

$$
W(T)=2 \int_{0}^{H} \delta\left(H^{\prime}, T\right) d H^{\prime}
$$


The inset in Figure 5 shows $\mathrm{W}$ as a function of temperature for a maximum magnetic field of $3 \mathrm{~T}$ for LPC-32. The magnetic work presents a very low value (almost zero) at temperatures above 225 $\mathrm{K}$, in the paramagnetic region at low magnetic signals. In the opposite sense, the low $\mathrm{W}$ values observed at low temperatures are consistent with the almost homogeneous low temperature ferromagnetic state. We observed high values in the intermediate temperature region, where the PS state is fully established; these high values could be associated with the nucleation of the CO phase, which competes with the FM phase, acting as pinning centers for the displacement of domain walls and the borders of the FM regions. Once the FM energy is enough to overcome the CO phase, the whole sample converts abruptly to the FM phase and no more extra W is needed.

The expression for the entropy change can be expressed as:

$\Delta S_{M}=\Delta S_{A N}-\frac{1}{2} \frac{\partial W}{\partial T}$

The expressions presented in equations 3 and 7 consider the entropy change calculated using Maxwell's relation for the case of an inhomogeneous and hysteretic system. Note that in the case of a homogeneous system, the FM fraction is constant below the critical temperature and no hysteresis appears in the magnetization curves, making equations 3 and 7 turn into $\Delta S_{H}$.

Taking into account the scenario described above, we will attempt to use it to explain the large difference between $\Delta S$ obtained from MR and from heat capacity measurements. In that sense we collapsed the abovementioned contributions in the following expression:

$\Delta S_{M}^{*}\left(T_{i}\right)=\Delta S_{H C}(T)+\alpha f(T)-\beta \frac{\partial W}{\partial T}(T)$

In order to find the maximum likelihood between $\Delta S_{M}^{*}$ and $\Delta S_{M}$, we studied the statistical distance between both expressions. The residual $r$ at a given temperature $T_{i}$ can be defined as 
$r_{\alpha \beta}\left(T_{i}\right)=\Delta S_{H C}\left(T_{i}\right)+\alpha f\left(T_{i}\right)-\beta \frac{\partial W}{\partial T}\left(T_{i}\right)-\Delta S_{M}\left(T_{i}\right)$

The estimation of $\alpha$ and $\beta$ can be obtained by minimizing the function

$\xi(\alpha, \beta)=\sum_{i} r_{\alpha \beta}^{2}\left(T_{i}\right)$

being $i$ the index for the measured point (in our case, between $50 \mathrm{~K}$ and $300 \mathrm{~K}$ ).

Numerically evaluating $\xi$ for $\alpha$ between -1 and 4 (with a step of 0.01 ) and $\beta$ between -20 to 50 (with a step of 0.1), we reached the minimum values for

$\alpha=(10.8 \pm 0.1) \frac{J}{K g K}$ and

$\beta=(0.46 \pm 0.01)$

It is interesting to note that the value obtained for $\alpha$ is close to the maximum entropy change obtained for the sample with $y=0.2(9 \mathrm{~J} / \mathrm{KgK})$, corresponding to the full FM sample. Additionally, the value obtained for parameter $\beta$ is close to the value predicted in equation $5(0.5)$.

In figure 6 we compare the temperature dependence of the entropy change measured using MR and $\mathrm{HC}$ and the one calculated from the optimized parameters in equation 8 . It can be observed that there is a very good agreement between the results obtained from MR and those extracted by using equation 8 .

\section{Conclusions}

Summarizing, in this paper we studied the influence of the phase separation phenomenon in magnetocaloric properties in samples of the $\mathrm{La}_{0.625-\mathrm{y}} \mathrm{Pr}_{\mathrm{y}} \mathrm{Ca}_{0.375} \mathrm{MnO}_{3}$ family. To do that we used three different samples (a FM homogeneous (LPC-20), a CO/AFM (LPC-50) and a phase separated (LPC-32)). Comparing the results obtained using two alternative experimental methods to 
calculate the entropy change, we observed a good agreement between both methods for the homogeneous samples. However, a great difference between both methods was observed for the phase separated sample. In order to understand the origin of this discrepancy, we improved the model by including two additional terms to the entropy change, which consider the effect of an inhomogeneous state and the hysteresis loops present at the $M(H)$ curves. Considering these two contributions, it was possible to reach the entropy change using MR, and taking the heat capacity entropy change as the starting point.

\section{Acknowledgments}

This paper has been possible thanks to the support from ANPCYT PICT 1506/2012 and 2016/2014. We wish to thank L. Granja for reading the manuscript and I. Urrutia and L. Fernandez Piana for their contributions in fruitful discussions. M.Q. is also a member of CIC-CONICET. 


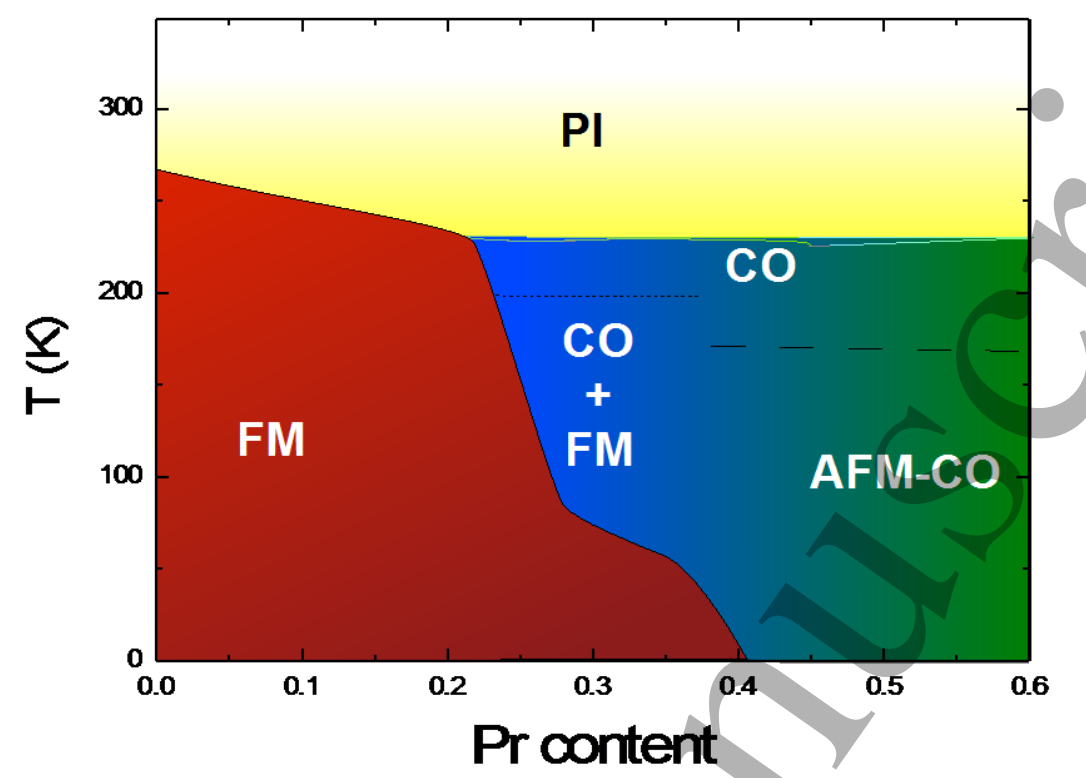

Figure 1: Magnetic phase diagram of $\mathrm{La}_{0.625-\mathrm{y}} \mathrm{Pr}_{\mathrm{y}} \mathrm{Ca}_{0.375} \mathrm{MnO}_{3}$ (FM: ferromagnetic metallic, $\mathrm{CO}$ : charge ordered, PI: paramagnetic insulator and AFM: antiferromagnetic).
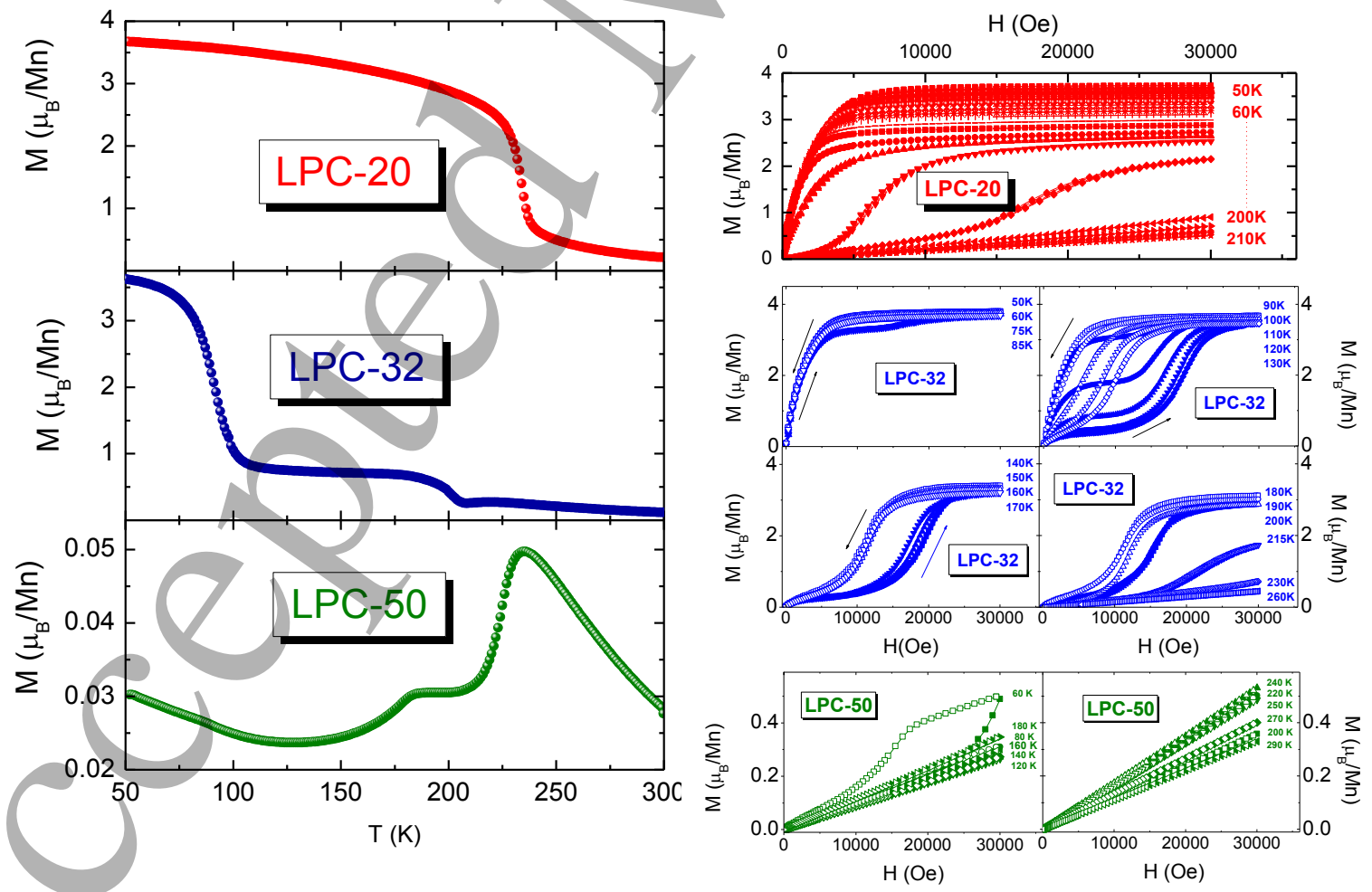
Figure 2: Magnetization as a function of temperature (left) and as a function of magnetic field(right) for samples $\mathrm{La}_{0.625-\mathrm{y}} \mathrm{Pr}_{\mathrm{y}} \mathrm{Ca}_{0.375} \mathrm{MnO}_{3}$ with $y=0.2$ (upper panel), $y=0.32$ (middle panel) and $y=0.5$ (lower panel). Solid (open) symbols indicate that the magnetic field is increasing (decreasing). Magnetization as a function of the magnetic field curves (right) for samples with $y=0.2$ (upper), $y=0.32$ (middle) and $\mathrm{y}=0.5$ (lower). The temperature of the curves are indicated.

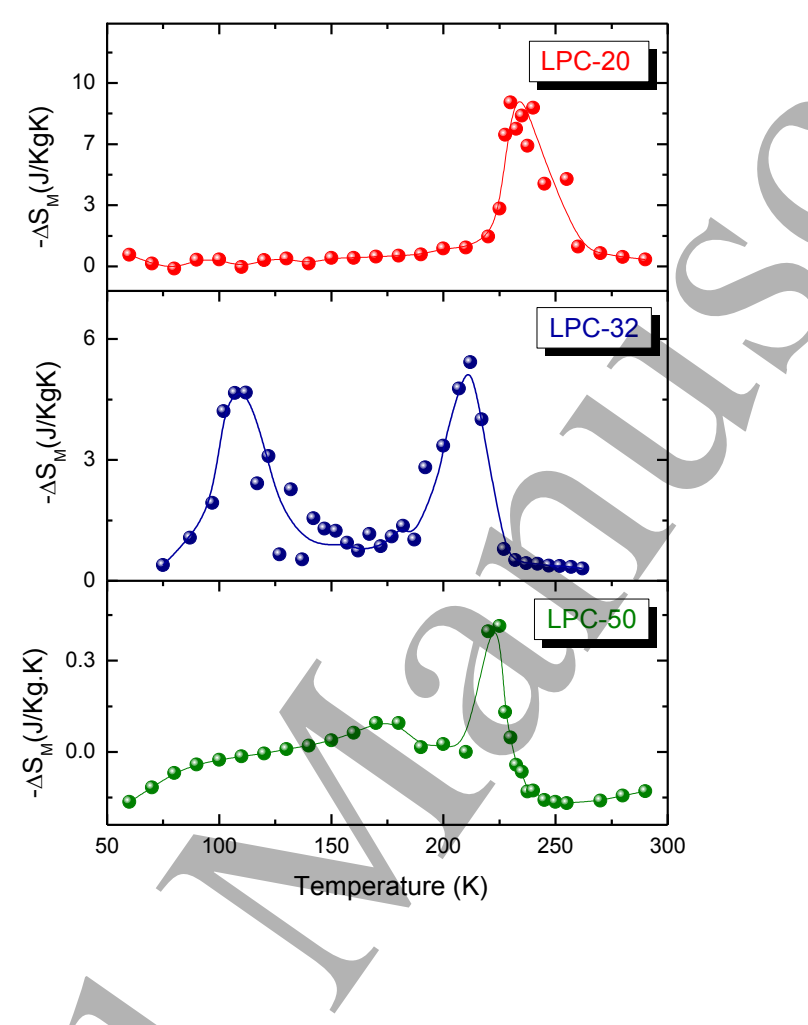

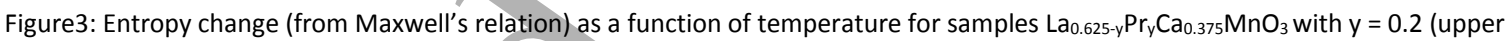
panel), $\mathrm{y}=0.32$ (middle panel) and $\mathrm{y}=0.5$ (lower panel). 

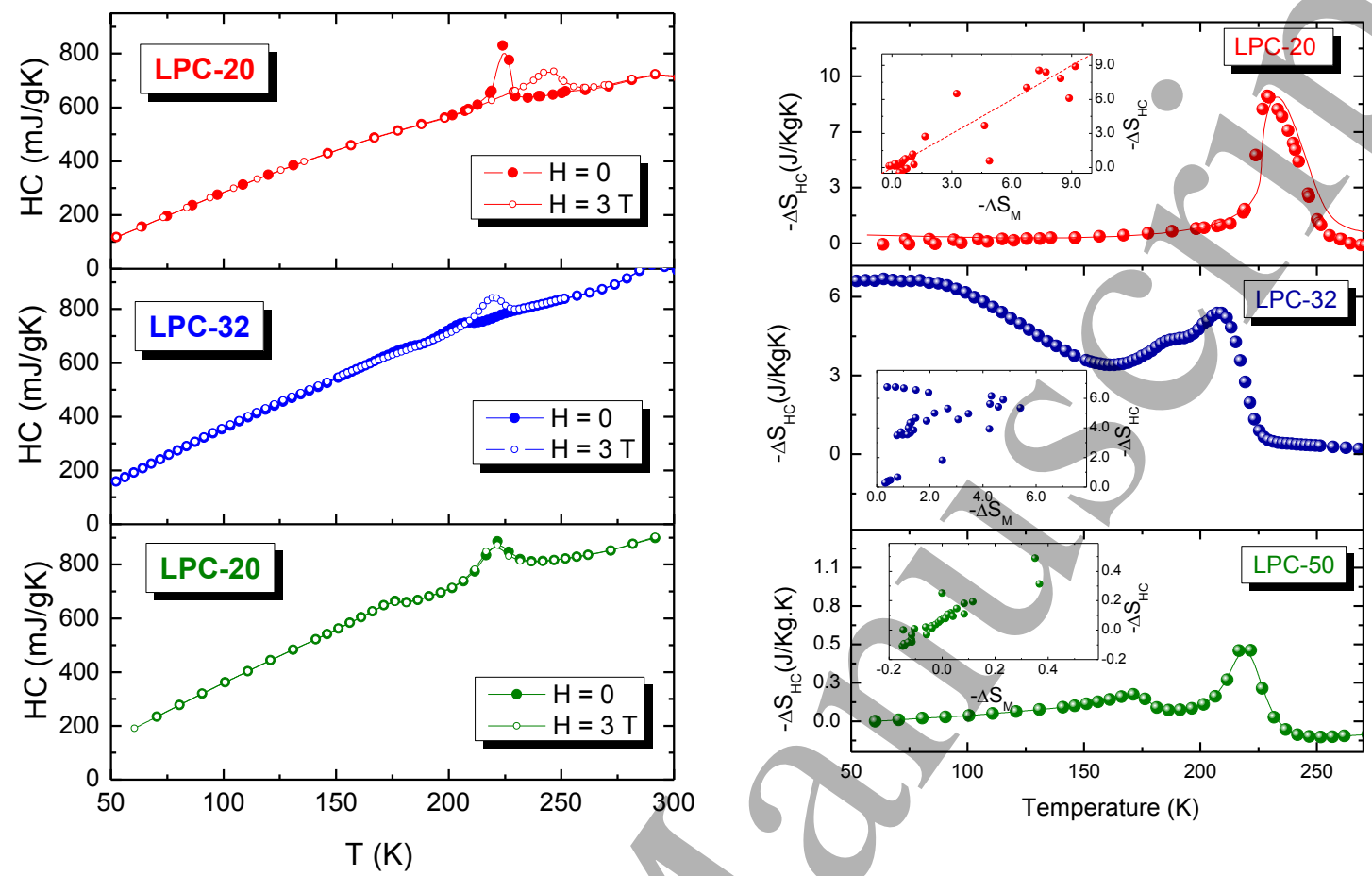

Figure 4: (Left) Heat capacity as a function of temperature and (right) entropy change (from heat capacity measurements) as a function of temperature for samples La.625-y $\mathrm{Pr}_{\mathrm{y}} \mathrm{Ca}_{0.375} \mathrm{MnO}_{3}$ with $\mathrm{y}=0.2$ (upper panel), $\mathrm{y}=0.32$ (middle panel) and $\mathrm{y}=0.5$ (lower panel). In the insets, $\Delta S_{M}$ and $\Delta S_{H C}$ are compared.

Figure 5: Schematic representation of an isothermal magnetization loop, where $M \uparrow$ and $M \downarrow$ are the two branches corresponding to increases and decreases in the magnetic field. MAN. The inset shows the temperature dependence of the magnetic work (W) defined as

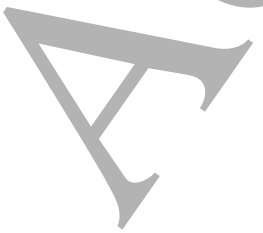
the area enclosed between $M \uparrow$ and $M \downarrow$ for the sample with y $=0.32$ 


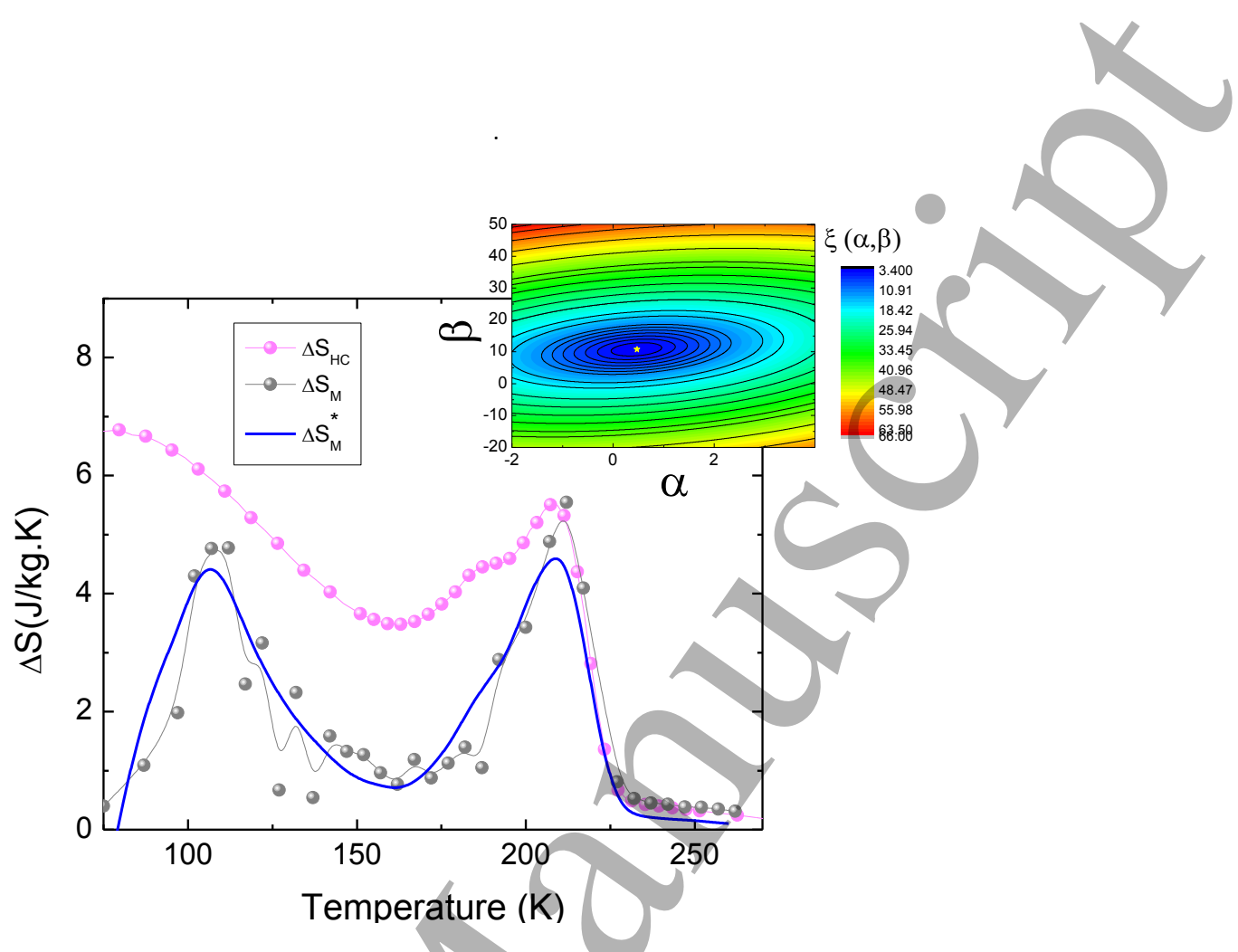

Figure 6: Entropy change as a function of temperature extracted from magnetization curves (gray), from heat capacity (pink) and from equation 8 (blue) for sample with $y=0.32$. The inset shows a color map of the error function $\xi$ used to obtain optimized $\alpha$ and $\beta$ values. 
[1] Weiss, Pierre and Piccard, Auguste, "Le phénomène magnétocalorique," J. Phys. Theor. Appl., vol. 7, no. 1, pp. 103-109, 1917.

[2] V. K. Pecharsky and K. A. Gschneidner Jr., "Giant Magnetocaloric Effect in Gd5 Si2 Ge2," Phys. Rev. Lett., vol. 78, no. 23, pp. 4494-4497, Jun. 1997.

[3] H. Zeng, J. Zhang, C. Kuang, and M. Yue, "Magnetic entropy change in bulk nanocrystalline Gd metals," Appl. Nanosci., vol. 1, no. 1, pp. 51-57, May 2011.

[4] H. Wada and Y. Tanabe, "Giant magnetocaloric effect of MnAs1-xSbx," Appl. Phys. Lett., vol. 79, no. 20, pp. 3302-3304, 2001.

[5] A. Planes, L. Mañosa, X. Moya, T. Krenke, M. Acet, and E. F. Wassermann, "Magnetocaloric effect in Heusler shape-memory alloys," J. Magn. Magn. Mater., vol. 310, no. 2, Part 3, pp. 2767-2769, 2007.

[6] C. S. Mejía, A. M. Gomes, and L. A. S. de Oliveira, "A less expensive NiMnGa based Heusler alloy for magnetic refrigeration," J. Appl. Phys., vol. 111, no. 7, p. 07A923, 2012.

[7] M. H. Phan and S. C. Yu, "Review of the magnetocaloric effect in manganite materials," J. Magn. Magn. Mater., vol. 308, no. 2, pp. 325-340, 2007.

[8] A. Rebello, V. B. Naik, and R. Mahendiran, "Large reversible magnetocaloric effect in La0.7xPr xCa0.3MnO3," J. Appl. Phys., vol. 110, no. 1, pp. 1-7, 2011.

[9] J. Pecharsky VK, Gschneidner KA, "Magnetocaloric effect from indirect measurements: magneti- zation and heat capacity.," J. Appl. Phys., vol. 86, no. 1999, pp. 565-75, 1999.

[10] M. Földeàki, R. Chahine, T.K. Bose, and M. Fiildeiki, "Magnetic measurements : A powerful tool in magnetic refrigerator design measurements : A powerful tool in magnetic refrigerator design," vol. 3528, no. May 2013, 1995.

[11] N. A. de Oliveira and P.J. von Ranke, "Magnetocaloric effect around a magnetic phase transition," Phys. Rev. B, vol. 77, no. 21, p. 214439, Jun. 2008.

[12] A. M. G. Carvalho, A. A. Coelho, P. J. von Ranke, and C. S. Alves, "The isothermal variation of the entropy $(\triangle S T)$ may be miscalculated from magnetization isotherms in some cases: MnAs and Gd5Ge2Si2 compounds as examples," J. Alloys Compd., vol. 509, no. 8, pp. 34523456, 2011.

[13] W. Cui, W. Liu, and Z. Zhang, "The origin of large overestimation of the magnetic entropy changes calculated directly by Maxwell relation," Appl. Phys. Lett., vol. 96, no. 22, pp. 1-4, 2010.

[14] G. Porcari et al., "Convergence of direct and indirect methods in the magnetocaloric study of first order transformations: The case of Ni-Co-Mn-Ga Heusler alloys," Phys. Rev. B Condens. Matter Mater. Phys., vol. 86, no. 10, pp. 1-5, 2012. 
[15] J. S. Amaral and V. S. Amaral, "On estimating the magnetocaloric effect from magnetization measurements," J. Magn. Magn. Mater., vol. 322, no. 9-12, pp. 1552-1557, 2010.

[16] L. Caron, Z. Q. Ou, T. T. Nguyen, D. T. Cam Thanh, O. Tegus, and E. Brück, “On the determination of the magnetic entropy change in materials with first-order transitions," $J$. Magn. Magn. Mater., vol. 321, no. 21, pp. 3559-3566, 2009.

[17] J. R. Sun, F. X. Hu, and B. G. Shen, "Comment on 'Direct Measurement of the 'Giant' Adiabatic Temperature Change in Gd5 Si2 Ge2," Phys. Rev. Lett., vol. 85, no. 19, p. 4191, Nov. 2000.

[18] G. J. Liu et al., "Determination of the entropy changes in the compounds with a first-order magnetic transition," Appl. Phys. Lett., vol. 90, no. 3, p. 32507, 2007.

[19] A. Giguère et al., "Direct Measurement of the 'Giant ' Adiabatic Temperature Change in Gd 5 Si 2 Ge 2," pp. 11-14, 1999.

[20] L. Caron, N. Ba Doan, and L. Ranno, "On entropy change measurements around first order phase transitions in caloric materials," J. Phys. Condens. Matter, vol. 29, no. 7, 2017.

[21] P. Levy et al., "Controlled phase separation in La0.5 Ca0.5 Mn O3," Phys. Rev. B, vol. 62, no. 10, pp. 6437-6441, Sep. 2000.

[22] A. Shahee and N. P. Lalla, "Oxygen stoichiometry: A key parameter to tune structural phase diagram of La0.2Sr0.8MnO3- $\delta$," AIP Conf. Proc., vol. 1665, no. 1, p. 30005, 2015.

[23] R. S. Freitas, L. Ghivelder, P. Levy, and F. Parisi, "Magnetization studies of phase separation in La0.5 Ca0.5 Mn O3," Phys. Rev. B, vol. 65, no. 10, p. 104403, Feb. 2002.

[24] Q. Huang et al., "Temperature and field dependence of the phase separation, structure, and magnetic ordering in La1-xCaxMnO3 ( $\mathrm{x}=0.47,0.50$, and 0.53)," Phys. Rev. B, vol. 61, no. 13, pp. 8895-8905, 2000 .

[25] J. Mira, J. Rivas, L. E. Hueso, F. Rivadulla, and M. A. López Quintela, “Drop of magnetocaloric effect related to the change from first- to second-order magnetic phase transition in La2/3(Ca1-xSrx)1/3MnO3," J. Appl. Phys., vol. 91, no. 10 I, pp. 8903-8905, 2002.

[26] F. Rivadulla et al., "Coexistence of paramagnetic-charge-ordered and ferromagneticmetallic phases in La0.5Ca0.5MnO3 evidenced by electron spin resonance," J. Appl. Phys., vol. 91, no. 2, pp. 785-788, 2002.

[27] M. Kim et al., "Raman scattering studies of temperature- and field-induced melting of charge order in (La,Pr,Ca)MnO3," pp. 1-13, 2008.

[28] C. H. Chen and S.-W. Cheong, "Commensurate to Incommensurate Charge Ordering and Its Real-Space Images in La0.5\$Ca0.5MnO3," Phys. Rev. Lett., vol. 76, no. 21, pp. 4042-4045, May 1996.

[29] S. Mori, C. H. Chen, and S.-W. Cheong, "Paired and Unpaired Charge Stripes in the Ferromagnetic Phase of La0.5 Ca0.5 Mn O3," Phys. Rev. Lett., vol. 81, no. 18, pp. 39723975, Nov. 1998. 
[30] T. Sarkar, B. Ghosh, A. K. Raychaudhuri, and T. Chatterji, "Crystal structure and physical properties of half-doped manganite nanocrystals of less than 100-nm size," Phys. Rev. B Condens. Matter Mater. Phys., vol. 77, no. 23, 2008.

[31] M. Uehara, S. Mori, C. H. Chen, and S.-W. Cheong, "Percolative phase separation underlies colossal magnetoresistance in mixed-valent manganites," Nature, vol. 399, p. 560, Jun. 1999.

[32] W. Wu, C. Israel, N. Hur, S. Park, S.-W. Cheong, and A. de Lozanne, "Magnetic imaging of a supercooling glass transition in a weakly disordered ferromagnet," Nat. Mater., vol. 5, p. 881, Oct. 2006.

[33] W. Kundhikanjana et al., "Direct Imaging of Dynamic Glassy Behavior in a Strained Manganite Film," Phys. Rev. Lett., vol. 115, no. 26, pp. 1-5, 2015.

[34] M. Quintero, S. Passanante, I. Irurzun, D. Goijman, and G. Polla, "Grain size modification in the magnetocaloric and non-magnetocaloric transitions in La0.5 CaO.5 Mn O3 probed by direct and indirect methods," Appl. Phys. Lett., vol. 105, no. 15, 2014.

[35] M. Quintero, J. Sacanell, L. Ghivelder, A. M. Gomes, A. G. Leyva, and F. Parisi, "Magnetocaloric effect in manganites: Metamagnetic transitions for magnetic refrigeration," Appl. Phys. Lett., vol. 97, no. 12, 2010.

[36] M. H. Phan, M. B. Morales, N. S. Bingham, H. Srikanth, C. L. Zhang, and S. W. Cheong, "Phase coexistence and magnetocaloric effect in La5/8-y Pry Ca3/8 Mn 03," Phys. Rev. B, vol. 81, no. 9, p. 94413, 2010.

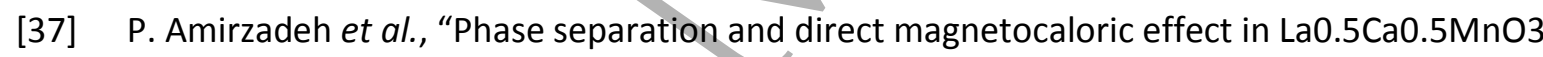
manganite," J. Appl. Phys., vol. 113, no. 12, p. 123904, 2013.

[38] J. M. D. Coey, M. Viret, andS. von Molnár, "Mixed-valence manganites - ten years on," Adv. Phys., vol. 58, no. 6, pp. 567-569, Nov. 2009.

[39] Z. Jirák, S. Krupička, Z. Šimša, M. Dlouhá, and S. Vratislav, "Neutron diffraction study of Pr1 - xCaxMnO3 perovskites," J. Magn. Magn. Mater., vol. 53, no. 1, pp. 153-166, 1985.

[40] L. Ghivelder et al., "Abrupt field-induced transition triggered by magnetocaloric effect in phase-separated manganites," Phys. Rev. B - Condens. Matter Mater. Phys., vol. 69, no. 21, 2004.

[41] A. L. L. Sharma, P.A. Sharma, S. K. McCall, S. B. Kim, and S. W. Cheong, "Enhanced magnetic refrigeration capacity in phase separated manganites," Appl. Phys. Lett., vol. 95, no. 9, pp. 95-97, 2009.

[42] D. C. Jiles and D. L. Atherton, "Theory of the magnetisation process in ferromagnets and its application to the magnetomechanical effect," J. Phys. D. Appl. Phys., vol. 17, no. 6, pp. 1265-1281, 1984. 